\title{
ADOPCIÓN DE PRÁCTICAS DE GOBIERNO CORPORATIVO DEL MERCADO CHILENO \\ EN EL TRIENIO 2015 - 2017
}

\section{ADOPTION OF CORPORATE GOVERNANCE PRACTICES OF THE CHILEAN MARKET IN TRIENNIUM 2015 - 2017}

\author{
Felipe Arenas Torres \\ Facultad de Economía y Negocios \\ Universidad de Talca \\ Talca, Chile \\ farenas@utalca.cl
}

\author{
Roberto Campos Troncoso \\ Facultad de Economía y Negocios \\ Universidad de Talca \\ Talca, Chile \\ rcampos@utalca.cl
}

\author{
Valentín Santander Ramírez \\ Facultad de Economía y Negocios \\ Universidad de Talca \\ Talca, Chile \\ Valentin.santander@utalca.cl
}

Fecha de recepción: 21/03/2019- Fecha de aprobación: 15/05/2019

\section{RESUMEN}

La presente investigación describe el grado de adopción de las prácticas de gobiernos corporativos de 203 sociedades anónimas abiertas chilenas, durante el trienio 2015 - 2017, en cumplimiento de la Norma de Carácter General N385, las mencionadas prácticas deben ser reportadas anualmente al regulador del mercado de valores en Chile, llamado Comisión para el Mercado Financiero. Los datos fueron analizados dentro de un intervalo de confianza del 95\%, dividiéndose en una primera etapa en un análisis estadístico descriptivo de los datos, y en una segunda parte presentando los niveles de adopción a nivel de mercado, categorías y principios para los períodos 2015, 2016 y 2017. En ambas etapas, se estableció que el grado de adopción es bajo (cercano al 30\%) y que en los tres años de estudio no se ha evidenciado interés por parte de las sociedades en generar avances significativos en la adopción de prácticas de gobierno corporativo.

PALABRAS CLAVE: Gobierno Corporativo; Adopción de prácticas; NCG №385.

\section{ABSTRACT}

The present research describes the degree of adoption of corporate governance practices of 203 Chilean open anonymous companies during the three-year 2015-2017, in

\footnotetext{
"Visión de Futuro" Año 16, Volumen N²3 N², Julio - Diciembre 2019 - Pág. 37 - 55

URL de la Revista: http://visiondefuturo.fce.unam.edu.ar/index.php/visiondefuturo/index

URL del Documento: http://visiondefuturo.fce.unam.edu.ar/index.php/visiondefuturo/issue/view/16

ISSN 1668 - 8708 - Versión en Línea

E-mail: revistacientifica@fce.unam.edu.ar
} 
compliance with Rule of General Character $N^{\circ} 385$, the aforementioned practices must be reported annually to the securities market regulator, called the Commission for the Financial Market. The data analysis, within a confidence interval of $95 \%$, was divided into two stages. First, with a descriptive statistical analysis, and secondly, the presentation of the levels of adoption at the market level, categories and principles for the periods 2015, 2016 and 2017. In both stages, it was established that the degree of adoption is low (close to $30 \%$ ), and that in the three years of study no interest to make significant improvement in the adoption of corporate governance practices has been evidenced by the corporations.

KEYWORDS: Corporate Governance; Degree of adoption; NCG №385.

\section{INTRODUCCIÓN}

Tras las crisis de desconfianza que viven diferentes mercados latinoamericanos producto de escándalos contables acontecidos en la última década, y de los que Chile no ha quedado atrás (Moraga \& Ropero, 2018), es perceptible que el concepto de gobiernos corporativos (GC) no se encuentra internalizado del todo por las sociedades anónimas abiertas chilenas.

En relación al punto anterior, tras la derogación, en el año 2015, de la Norma de Carácter General №341 (NCG №341) de gobiernos corporativos, después de estar vigente por un período de tres años, la Superintendencia de Valores y Seguros, actual Comisión para el Mercado Financiero (CMF), observó que la normativa no cumplía con sus expectativas, debido a que las respuestas entregadas por las sociedades eran muy acotadas, no se entregaba mayores antecedentes y tampoco las entidades adoptaban prácticas diferentes a las propuestas por el regulador (Superintendencia de Valores y Seguros, 2015). A raíz de lo anterior, en el año 2015 se inició un proyecto de fortalecimiento de los estándares de gobierno corporativo, que derivó en la vigente Norma de Carácter General N`385 (NCG N`385).

Al respecto, la CMF, como regulador del mercado financiero en Chile, al momento de implementar la nueva normativa, buscó subsanar dos debilidades de la NCG N³41, una de ellas, el cumplimiento formal, pero no práctico, de lo exigido por la normativa vigente de GC y la ausencia de interés, por parte de las entidades, en mejorar sus prácticas (Superintendencia de Valores y Seguros, 2015). En el primer caso, se especificó en el cuerpo normativo lo que se entenderá por práctica adoptada, mientras que en el segundo, es el inversionista quién debiera influir directa o indirectamente en el aumento del grado de adopción.

En la misma línea, al considerar el grado de adhesión a las prácticas de GC, estudios relacionados, señalan que el cumplimiento normativo de las empresas emisoras de valores

\footnotetext{
"Visión de Futuro" Año 16, Volumen N²3 N², Julio - Diciembre 2019 - Pág. 37 - 55

URL de la Revista: http://visiondefuturo.fce.unam.edu.ar/index.php/visiondefuturo/index

URL del Documento: http://visiondefuturo.fce.unam.edu.ar/index.php/visiondefuturo/issue/view/16

ISSN 1668 - 8708 - Versión en Línea

E-mail: revistacientifica@fce.unam.edu.ar
} 
de oferta pública respecto a la NCG N³85, asciende a un promedio del $31 \%$ para el año 2015 (PwC Chile y ESE Business School Universidad de los Andes, 2016), inclusive entre el año 2015 y 2016 el grado de adopción aumentó un 3\%, esto representa un avance marginal a lo esperado luego de que las compañías tuvieran un año más para fortalecer su estructura de gobiernos corporativos y responsabilidad social (Arenas \& Sánchez, 2017).

Existen algunas investigaciones en la temática ligadas al ámbito académico profesional, por ejemplo Moraga y Ropero (2018) en su estudio realizan un análisis correlacional entre el grado de adopción de prácticas de la NCG ํ385 y la rentabilidad sobre las ventas de las empresas más importantes del mercado bursátil chileno durante el año 2015, concluyendo que no se ha establecido una relación, debido al desconocimiento de la normativa y la implementación de sus recomendaciones.

Por su parte, firmas consultoras realizan análisis descriptivos, presentando el grado de adopción promedio de las sociedades que reportaron. Por ejemplo, Iltis presenta el grado de adopción de las empresas para los períodos 2015, 2016 y 2017 (Iltis Consulting, 2019). En la misma línea de análisis, PwC Chile en conjunto con la Universidad de los Andes (2016) realiza un estatus de los directorios en Chile considerando la información de la NCG №385 reportada al 31 de diciembre 2015, al igual que la empresa A\&C Consultores Auditores (A\&C Consultores Auditores, 2019). Determinando los tres análisis, que el grado de adopción es cercano a un tercio de la propuesta y que la adhesión a las recomendaciones es dispar al comparar categorías, principios y prácticas.

De acuerdo a lo anterior, el gobierno corporativo no debe considerarse como un tema de moda, sino como la solución a la crisis de confianza que se percibe alrededor de las empresas (Castro \& Cano, 2004), aún más, cuando existe desconocimiento generalizado en los diversos stakeholders sobre las prácticas recomendadas por el regulador en la materia (Moraga \& Ropero, 2018). En este sentido, el propósito de esta investigación consiste en describir el grado de adopción de las prácticas de gobierno en el período 2015 - 2017, entregando una mirada académica, que permita detectar aquellas categorías y principios que poseen un menor grado de adhesión. 


\section{DESARROLLO}

\section{El gobierno corporativo como mecanismo de auto-regulación}

Para describir el grado de adopción de las prácticas de GC en primera instancia es importante conocer sus fundamentos, la evolución del marco regulatorio vigente en Chile y la literatura científica relacionada a los beneficios de adoptar prácticas de gobernanza corporativa.

En los últimos años se ha observado una proliferación de iniciativas que proponen establecer guías y lineamientos específicos que permitan aplicar prácticas de gobernanza (Superintendencia de Valores y Seguros, 2015), materializándose ya en el año 2009 en 2.147 publicaciones sobre un buen gobierno empresarial en la base de datos ISI Web of Science con 79.635 referencias respectivamente (Richart-Ramón et al, 2011).

Adicionalmente en la temática existe un consenso respecto a que las deficiencias en el GC han jugado un papel muy relevante en la génesis y el desarrollo de las crisis financieras, esto sumado a la interconexión mundial en los mercados de capitales, hacen indispensable la armonización de un marco internacional de prácticas de gobierno (Fundación Instituto Iberoamericano de Mercado de Valores, 2015), incorporando factores propios de cada país donde se aplique la normativa.

En el rol de regulador, es necesario que el Estado desempeñe una función controladora del mercado de valores, debido a que es difícil sostener que los mercados resuelvan sus problemas de gobierno de las corporaciones por si solos y es complejo igualmente sostener que las propias corporaciones sean capaces de autorregularse (Agüero, 2009).

Respecto al concepto de GC, este abarca una serie de relaciones entre el cuerpo directivo de una empresa, su Consejo, sus accionistas y otras partes interesadas (Organización para la Cooperación y el Desarrollo Económico, 2004), entendiéndose como los métodos mediante los cuales se establece orden en las empresas, y asegura que en el proceso de toma de decisiones los intereses grupales se encuentren representados (Ruiz \& Steinwascher, 2007).

En relación al marco regulatorio vigente en Chile, este se estructura en dos partes, la primera corresponde a la legislación promulgada a través del Congreso Nacional, considerando la Ley de Mercado de Valores y la Ley de Sociedades Anónimas, ambas promulgadas en 1981, pero con dos importantes modificaciones en la materia. La Ley N¹9.705 que regula la oferta pública de adquisición de acciones (OPAS) promulgada en el año 2000, y la Ley №20.382, llamada Ley de Gobiernos Corporativos, la cual introduce un perfeccionamiento a la normativa que regula la gobernanza al interior de las empresas.

\footnotetext{
“Visión de Futuro" Año 16, Volumen N²3 N², Julio - Diciembre 2019 - Pág. 37 - 55

URL de la Revista: http://visiondefuturo.fce.unam.edu.ar/index.php/visiondefuturo/index

URL del Documento: http://visiondefuturo.fce.unam.edu.ar/index.php/visiondefuturo/issue/view/16 
La segunda parte del marco regulatorio, considera la creación de la Superintendencia de Valores y Seguros, actual Comisión para el Mercado Financiero, a través del Decreto de Ley $N^{`} 3.580$ de 1980, organismo emisor de las normas para la difusión de información respecto a las prácticas de gobierno corporativo de las sociedades anónimas abiertas, promulgando en 2015 la NCG №385 en reemplazo de la NCG `o341.

En relación a la NCG $\mathrm{N} \times 385$, vigente actualmente en Chile, esta posee como característica que es una autoevaluación bajo el criterio Cumplir o Explicar, normando la difusión de información respecto de las prácticas de GC adoptadas por las sociedades anónimas abiertas chilenas, de una manera voluntaria, motivando la transparencia de las sociedades con sus diferentes stakeholders.

Respecto a lo que se entenderá por práctica adoptada, el regulador en el cuerpo normativo hizo una especificación a esto, señalando

"que la sociedad adopta una práctica determinada, cuando ella cuenta con políticas aprobadas, y los procedimientos, mecanismos y sistemas completamente implementados y en funcionamiento, para todos los elementos descritos en la respectiva práctica. En caso contrario, debe entenderse que no adopta la práctica, sin perjuicio que si es intención de la sociedad sea adoptarla" (Superintendencia Valores y Seguros, 2015, s/p).

En consideración a la estructura de la NCG N³85, esta se divide en cuatro categorías, 23 principios y 99 prácticas, atribuyendo un mayor número de principios y prácticas a la conformación y funcionamiento del Directorio, luego en nivel de recomendaciones propuestas se encuentra la relación con los accionistas, la gestión de riesgos y por último la autoevaluación del Directorio, el esquema se presenta en la Tabla №1.

Tabla $N^{\circ} 1$. Contenido de la NCG N³85

\begin{tabular}{lcr}
\hline \multicolumn{1}{c}{ Categoría } & Principios & Prácticas \\
\hline Funcionamiento y composición del Directorio & 11 & 51 \\
\hline $\begin{array}{l}\text { Relación entre la sociedad, los accionistas y el } \\
\text { público en general }\end{array}$ & 7 & 22 \\
\hline De la gestión y control de riesgos & 4 & 22 \\
\hline De la evaluación por parte de un tercero & 1 & 4 \\
\hline Total & 23 & 99 \\
\hline
\end{tabular}

Fuente: Elaboración Propia

Cabe agregar, que en relación a la legislación que regula el GC, Zegers \& Consiglio (2013), señalan que es importante que las nuevas normas no sean una mera reacción a los abusos y escándalos que ocurren en la vida real, agrega Rivero (2005), que estas deben fomentar el desarrollo de mejores prácticas de gobierno corporativo, de manera voluntaria y

\footnotetext{
“Visión de Futuro" Año 16, Volumen N²3 N², Julio - Diciembre 2019 - Pág. 37 - 55

URL de la Revista: http://visiondefuturo.fce.unam.edu.ar/index.php/visiondefuturo/index

URL del Documento: http://visiondefuturo.fce.unam.edu.ar/index.php/visiondefuturo/issue/view/16

ISSN 1668 - 8708 - Versión en Línea

E-mail: revistacientifica@fce.unam.edu.ar
} 
no ser sustituidos por normas imperativas, debido a que se estaría reconociendo fallos en el sistema, debilitando el potencial productivo y creativo de valor de la gestión empresarial.

Simultáneamente, los esfuerzos por generar normativa relacionada al tema, deben ir en línea con los principios de la OCDE, por tanto resultaría conveniente que dada la gran cantidad de reportes que realizan las entidades reguladas, los esfuerzos deberían concentrarse en definir un único documento que fuera fácil de consultar por los interesados en materia de GC (Briozzo et al, 2018).

Es evidente, entonces, que adoptar prácticas de GC influye en el desempeño empresarial, al respecto el contar con buenas prácticas de gobierno corporativo mejora la disponibilidad y las condiciones de obtención de recursos (Altamirano et al, 2019). Anteriormente Sanchez y García Meca (2004) estudiaron la influencia del GC en la rentabilidad de las empresas españolas, concluyendo que existe una influencia significativa en la rentabilidad financiera de las organizaciones, a partir de la adopción de estándares de gobierno corporativo, que según Paul (2017), al constituirse como un medio multidisciplinario de garantía y prevención que permite prevenir los problemas de fraude o mala gestión en la empresa, buscando el beneficio de los actores, brindando transparencia, sostenibilidad, supervisión y rendición de cuentas.

\section{Metodología}

El estudio es de carácter descriptivo - longitudinal, dado que en el primer caso se presentarán los resultados de la NCG №385, respecto a su grado de adopción, y es longitudinal dado que los datos de la población se obtendrán en distintos momentos durante el período 2015 - 2017. Por otra parte, la recolección de los datos se realizará a través del tipo fuentes documentales y estadísticas (Hueso \& Cascant, 2012), específicamente a través del banco de datos perteneciente a la CMF de Chile.

Cada directorio de una sociedad anónima abierta reporta a la CMF la adopción de la NCG N³85, en marzo del período siguiente al informado, la que se encuentra conformada por 99 recomendaciones que entrega el regulador y que deben anualmente ser reportadas al organismo sobre su adopción. El universo de este estudio fue de 203 sociedades que presentan reportes en cada año del trienio de análisis. Las respuestas en este sentido son de carácter dicotómica, indicando S como sí cumple con la práctica, o $\mathrm{N}$ como no ha adoptado la práctica, cabe destacar que para efectos del análisis de los datos, $\mathrm{S}=1$ y $\mathrm{N}=0$.

Luego se definió un intervalo de confianza del 95\% bajo una distribución normal, con el fin de analizar los datos desde una mirada académica, quedando en el primer caso una población delimitada a esta restricción de 196 sociedades para el año 2015, 200 para el año 2016 y 203 para el 2017.

\footnotetext{
“Visión de Futuro" Año 16, Volumen N²3 N², Julio - Diciembre 2019 - Pág. 37 - 55

URL de la Revista: http://visiondefuturo.fce.unam.edu.ar/index.php/visiondefuturo/index

URL del Documento: http://visiondefuturo.fce.unam.edu.ar/index.php/visiondefuturo/issue/view/16

ISSN 1668 - 8708 - Versión en Línea

E-mail: revistacientifica@fce.unam.edu.ar
} 
Para obtener este resultado se acotaron los datos en el intervalo de confianza especificado anteriormente, de acuerdo a lo utilizado por estudios de ciencias sociales. El procedimiento consistió primero en estandarizar los datos de acuerdo a: $Z=\frac{(X-\mu)}{\sigma}$ con el fin de comparar efectivamente los grados de adopción de prácticas de GC de las empresas en estudio (Anderson et al, 2008).

Una vez estandarizados, los valores que se obtuvieron y que se estudiaran, aparecen distribuidos normalmente dentro de $\pm 1,96$ desviaciones estándar de la media (estandarización del grado de adopción). Por lo tanto, los valores estudiados de $\overline{\mathrm{X}}$ estarán dentro de $\pm 1.96 \sigma_{\bar{x}}$ de la media $\mu$. Siendo la estimación del intervalo de la media poblacional $\mu, \overline{\mathrm{X}} \pm$ margen de error con $\sigma_{\overline{\mathrm{x}}}=\sigma / \sqrt{n}$.

\section{Resultados}

Los resultados se presentan en dos apartados, primero un análisis estadístico descriptivo del grado de adopción a las prácticas de la NCG №385 durante el período 2015 2017, considerando el alfa de Cronbach, la media, la desviación estándar y la varianza de los datos. En segundo lugar, se presenta el grado de adopción de la población estudiada a nivel promedio de las sociedades que reportan, incorporando la clasificación por categoría, principios y prácticas.

En primera instancia los resultados de la Tabla $N^{\circ} 2$ muestra la fiabilidad de los datos obtenidos a través del coeficiente Alfa de Cronbach, siendo en los tres períodos analizados superior a 0,9 lo que indica una fuerte consistencia interna entre las respuestas a las recomendaciones de la NCG N³85.

Tabla №2. Estadístico de fiabilidad respuestas a la NCG №385

\begin{tabular}{lrrr}
\hline Variables & \multicolumn{3}{c}{ Período 2015 Período 2016 Período 2017 } \\
\hline Número de empresas & 196 & 200 & 203 \\
\hline Número de prácticas & 99 & 99 & 99 \\
\hline Alfa de Cronbach & 0,948 & 0,962 & 0,968 \\
\hline
\end{tabular}

Fuente: Elaboración Propia

Al analizar los elementos estadísticos descriptivos de cada período, de acuerdo a la Tabla $N^{\circ} 3$, se puede apreciar que hubo sociedades que no adoptaron prácticas del estándar propuesto. Adicionalmente, el máximo de recomendaciones adoptadas dentro de una distribución normal aumento un 16\% dentro del período 2015 - 2017, mientras que la media

\footnotetext{
“Visión de Futuro" Año 16, Volumen N²3 N², Julio - Diciembre 2019 - Pág. 37 - 55

URL de la Revista: http://visiondefuturo.fce.unam.edu.ar/index.php/visiondefuturo/index

URL del Documento: http://visiondefuturo.fce.unam.edu.ar/index.php/visiondefuturo/issue/view/16

ISSN 1668 - 8708 - Versión en Línea

E-mail: revistacientifica@fce.unam.edu.ar
} 
de respuestas positivas fue para el año 2015 de un 28,35\% y para el período 2017 de 34,85\%, aumentando un $6,5 \%$.

Respecto a la desviación estándar y varianza, esta es de 16,06 y 257,77 para el primer año de evaluación, aumentado a 20,85 y 434,52 respectivamente para el período 2017, lo que significa el grado de variabilidad que tienen con respecto a la media, en relación a la adopción de las prácticas de gobierno corporativo.

Tabla $\mathbf{N}^{\circ} 3$. Estadísticos descriptivos respuestas positivas a la NCG $\mathbf{N}^{\circ} 385$

Estadísticos Descriptivos Período 2015 Período 2016 Período 2017

\begin{tabular}{lrrr}
\hline Mínimo & 0 & 0 & 0 \\
\hline Máximo & 77 & 90 & 93 \\
\hline Media & 28,35 & 32,59 & 34,85 \\
\hline Desviación Estándar & 16,06 & 19,09 & 20,85 \\
\hline Varianza & 257,77 & 364,25 & 434,52 \\
\hline
\end{tabular}

Fuente: Elaboración Propia

Después de analizar las 99 prácticas adoptadas entre el período de reporte 2015 y 2017 de la NCG N³85, de acuerdo al Tabla $N^{\circ} 4$, los resultados indican que existió un aumento en el grado de adopción total de un 6,6\%, siendo el promedio del último año de reporte un 35,2\%.

Tabla N4. Resultados comparativos NCG N³85 período 2015 - 2017

\begin{tabular}{lrrr}
\hline \multicolumn{1}{c}{ Categoría } & $\begin{array}{c}\text { G' Adopción } \\
\text { año } \mathbf{2 0 1 5}\end{array}$ & $\begin{array}{c}\text { Adopción } \\
\text { año } \mathbf{2 0 1 6}\end{array}$ & $\begin{array}{c}\text { Adopción } \\
\text { año 2017 }\end{array}$ \\
\hline Funcionamiento y composición del Directorio & $29,0 \%$ & $33,4 \%$ & $36,1 \%$ \\
\hline $\begin{array}{l}\text { Relación entre la sociedad, los accionistas y el } \\
\text { público en general }\end{array}$ & $26,2 \%$ & $29,1 \%$ & $30,7 \%$ \\
\hline De la gestión y control de riesgos & & & \\
\hline De la evaluación por parte de un tercero & $34,7 \%$ & $40,0 \%$ & $42,1 \%$ \\
\hline
\end{tabular}

Fuente: Elaboración Propia

Respecto al nivel de avance por categorías, los resultados indican que el área relacionada al directorio y a la relación con los accionistas, se encuentran individualmente en un tercio de adhesión a la NCG `385, mientras que en materia de gestión y control de riesgos la adopción aumenta a un 42,1\%, siendo la más alta. Por último, la categoría correspondiente a la evaluación por un tercero independiente posee un 9,5\% de adhesión al estándar.

En relación a los principios por cada categoría, los resultados son dispares, por ejemplo en la composición y funcionamiento del directorio, de acuerdo a la Figura $\mathrm{N}^{\circ}{ }^{1}$, el principio que posee un mayor grado de adhesión es el relacionado a la inducción de nuevos integrantes, siendo para el 2017 de un $76 \%$, en contraste al 10\% de adopción del principio 1.g Reuniones

“Visión de Futuro" Año 16, Volumen No 23 N², Julio - Diciembre 2019 - Pág. 37 - 55

URL de la Revista: http://visiondefuturo.fce.unam.edu.ar/index.php/visiondefuturo/index

URL del Documento: http://visiondefuturo.fce.unam.edu.ar/index.php/visiondefuturo/issue/view/16

ISSN 1668 - 8708 - Versión en Línea

E-mail: revistacientifica@fce.unam.edu.ar 
con la unidad de responsabilidad social. Llama también la atención el grado de adopción de un $23 \%$ relacionado a los procedimientos de mejoramiento continuo que poseen las sociedades.

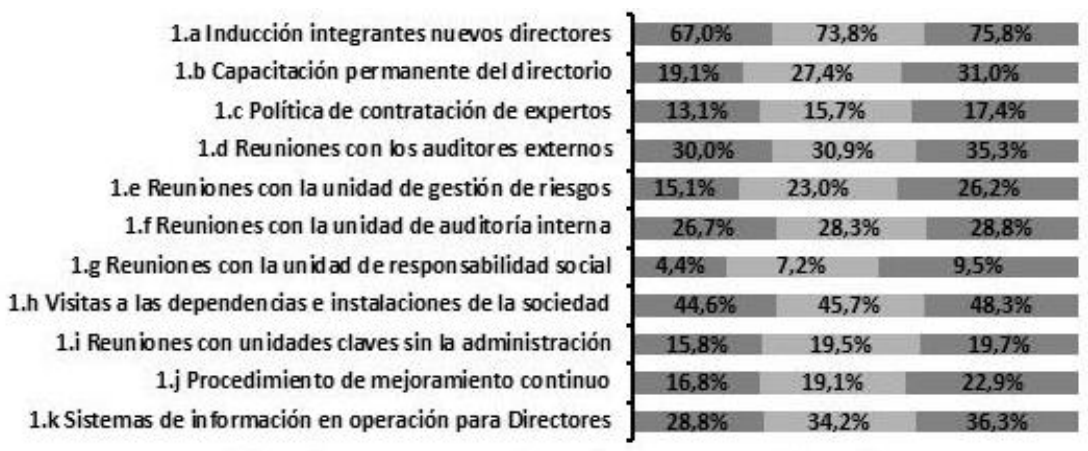

adopción 2015 adopción 2016 adopción 2017

Figura $\mathrm{N}^{\circledR} 1$. Grado de adopción categoría funcionamiento y composición del directorio

Fuente: Elaboración Propia

De acuerdo a la Figura $\mathrm{N}^{\circ} 2$, el $60 \%$ de las sociedades declaran tener unidades de relación con los accionistas y un $88 \%$ tiene una página web actualizada para ellos. Respecto al principio con menor grado de adopción en esta categoría, se encuentra la participación por medios remotos de accionistas en las juntas generales de accionistas.

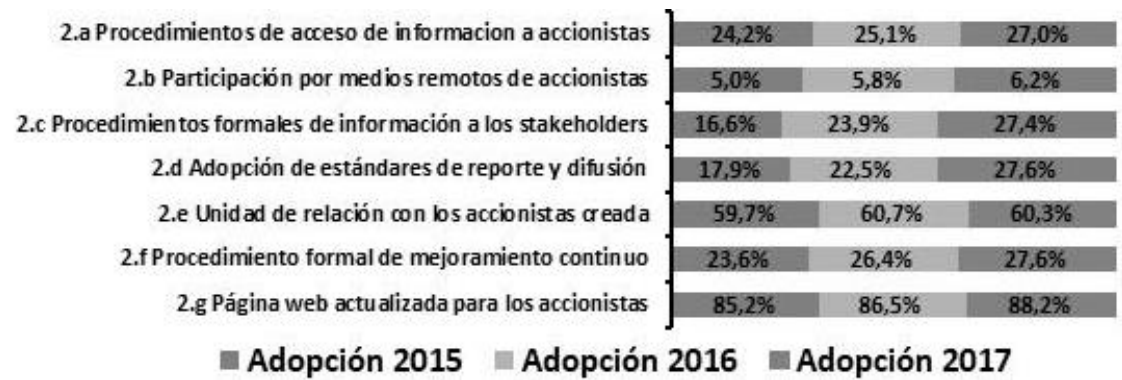

Figura N². Grado de adopción categoría relación entre la sociedad, los accionistas y el público en general

Fuente: Elaboración Propia

En relación a la categoría de la gestión y control de riesgos, la Figura N3 nos indica que uno de los principios que posee mayor grado de adopción, en el período analizado, es el establecimiento de canales de denuncias éticas y que éstos se encuentren vigentes, con un $63 \%$ para el año 2017. Mientras que tan solo un 19\% de las sociedades declaran tener aprobado procedimientos relacionados a la aprobación de las estructuras salariales de los altos ejecutivos de las sociedades.

\footnotetext{
"Visión de Futuro" Año 16, Volumen N²3 N², Julio - Diciembre 2019 - Pág. 37 - 55

URL de la Revista: http://visiondefuturo.fce.unam.edu.ar/index.php/visiondefuturo/index

URL del Documento: http://visiondefuturo.fce.unam.edu.ar/index.php/visiondefuturo/issue/view/16

ISSN 1668 - 8708 - Versión en Línea

E-mail: revistacientifica@fce.unam.edu.ar
} 


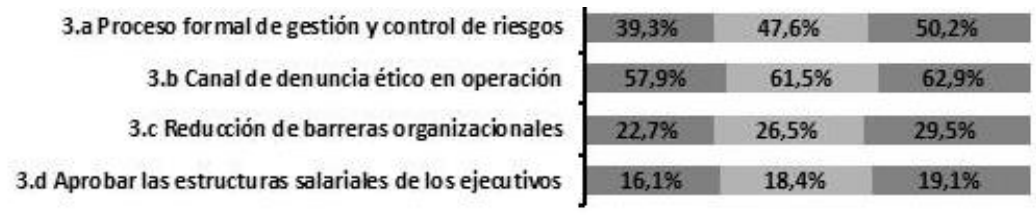

EAdopción 2015 Adopción 2016 Adopción 2017

Figura $\mathrm{N}^{\circledR} 3$. Grado de adopción de la gestión y control de riesgos

Fuente: Elaboración Propia

De acuerdo a la Figura $\mathrm{N}^{\circ} 4$, uno de los principios que posee un menor grado de adopción, se refiere a las prácticas enmarcadas dentro de la autoevaluación del directorio, siendo la adhesión de un 9,5\% para el año 2017, aumentando 5,4 puntos porcentuales en el período analizado.

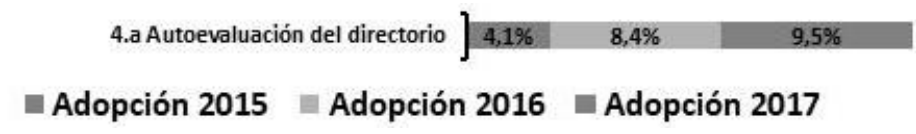

Figura $N^{\circ} 4$. Grado de adopción de la evaluación por parte de un tercero

Fuente: Elaboración Propia

A nivel de prácticas, al igual que en los principios de la normativa, los resultados son dispares y difieren de acuerdo a la temática abordada por el regulador, a continuación, se presentan los resultados de las recomendaciones más relevantes.

La Figura N5 muestra la adopción que tuvieron las prácticas relacionadas a los procedimientos definidos para la capacitación y actualización permanente del Directorio, entre ellas materias relacionadas a GC, inclusión, diversidad, sostenibilidad, gestión de riesgos, deberes y conflicto de intereses. Por ejemplo un 36,5\% de las sociedades el 2017 considera entre las capacitaciones del Directorio, el estudio de las mejores prácticas de GC adoptadas por diferentes empresas, mientras que solo un $27,6 \%$ considera los avances a nivel local e internacional en material de inclusión dentro de los procedimientos del Directorio.

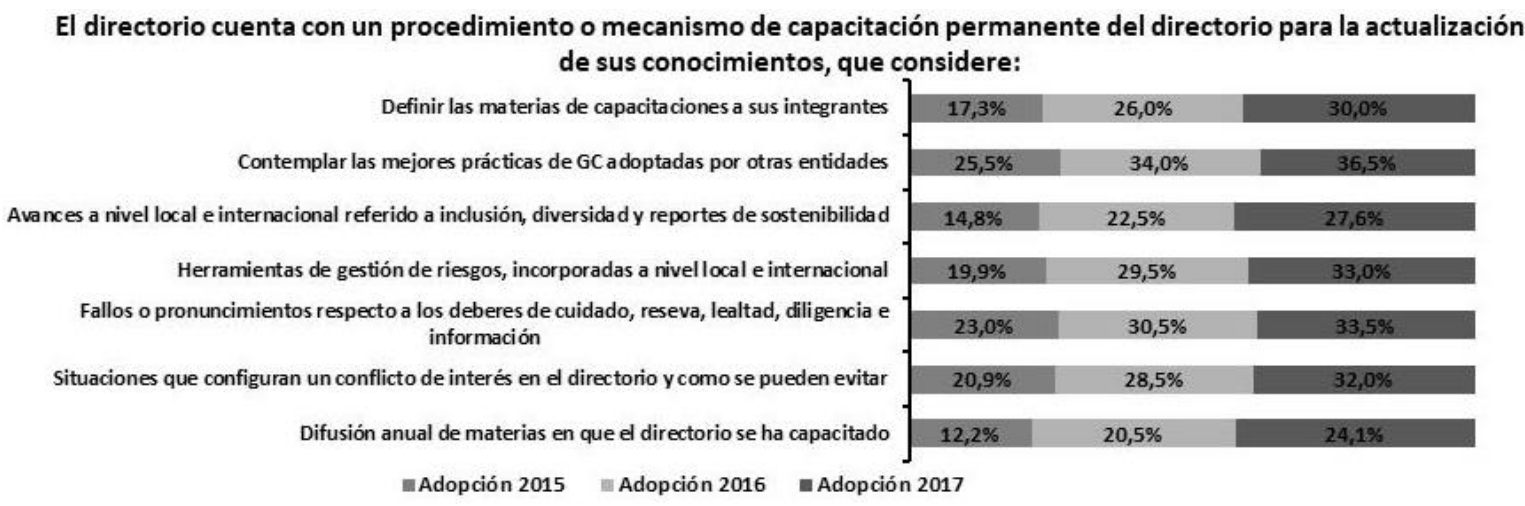

Figura №5. Grado de adopción por prácticas, principio 1.b

Fuente: Elaboración Propia

\footnotetext{
“Visión de Futuro" Año 16, Volumen N² 23 N², Julio - Diciembre 2019 - Pág. 37 - 55

URL de la Revista: http://visiondefuturo.fce.unam.edu.ar/index.php/visiondefuturo/index

URL del Documento: http://visiondefuturo.fce.unam.edu.ar/index.php/visiondefuturo/issue/view/16

ISSN 1668 - 8708 - Versión en Línea

E-mail: revistacientifica@fce.unam.edu.ar
} 
En cuanto a las políticas relacionadas a la contratación de expertos, la Figura $N^{\circledR} 6$ indica que existe un aumento marginal en la adhesión a las prácticas, y que un 17,7\% de las empresas posee una política que considere la contratación de una asesoría si esto es requerido por un integrante del Directorio. Respecto a la difusión de asesorías solicitadas y no contratadas, la adopción es de un 14,3\%, siendo la que más avanzo en este principio dentro del trienio analizado.

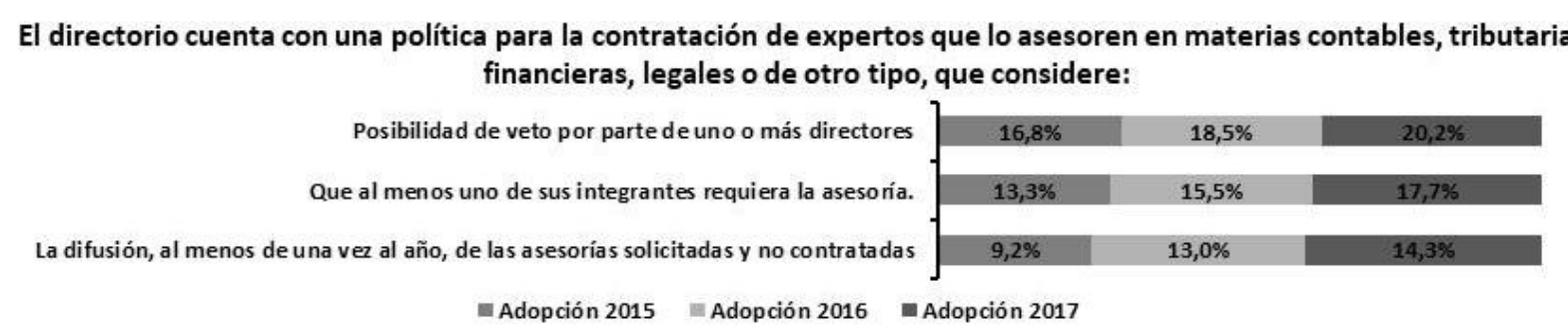

Figura $\mathbf{N}^{\circ} 6$. Grado de adopción por prácticas, principio 1.c

Fuente: Elaboración Propia

Otro grupo de recomendaciones relevantes, se refiere a si el directorio se reúne trimestralmente con la empresa de auditoría externa, con el departamento de gestión de riesgos y la unidad de auditoría interna. De acuerdo a la Figura $\mathrm{N}^{\circ} 7$, en primera instancia, el grado de adhesión promedio es de un 35\% para los revisores externos, en donde el análisis del plan de auditoría, las diferencias detectadas y sus resultados del proceso de revisión, tiene una adopción bastante homogénea.

Respecto a las reuniones con el área de gestión de riesgos, los resultados presentan un menor grado de adhesión, por ejemplo solo un $28,1 \%$ revisa la matriz de riesgos empleada por la unidad, y solo un 18,2\%, analiza los planes de contingencias, frente a eventos que afecten la continuidad del negocio. En el punto de la unidad de auditoría interna, la adopción es similar, en este sentido solo un $27,1 \%$ de los directorios se preocupa de analizar el plan de auditoría interna. Continuando con el análisis, por ejemplo el 28,1\% analiza la efectividad de los modelos de prevención de delitos implementados por la organización.

\footnotetext{
“Visión de Futuro" Año 16, Volumen N²3 N², Julio - Diciembre 2019 - Pág. 37 - 55

URL de la Revista: http://visiondefuturo.fce.unam.edu.ar/index.php/visiondefuturo/index

URL del Documento: http://visiondefuturo.fce.unam.edu.ar/index.php/visiondefuturo/issue/view/16

ISSN 1668 - 8708 - Versión en Línea

E-mail: revistacientifica@fce.unam.edu.ar
} 
El directorio se reune al menos trimestralmente con la empresa de auditoría externa a cargo de la auditoría de los estados financieros, para analizar:

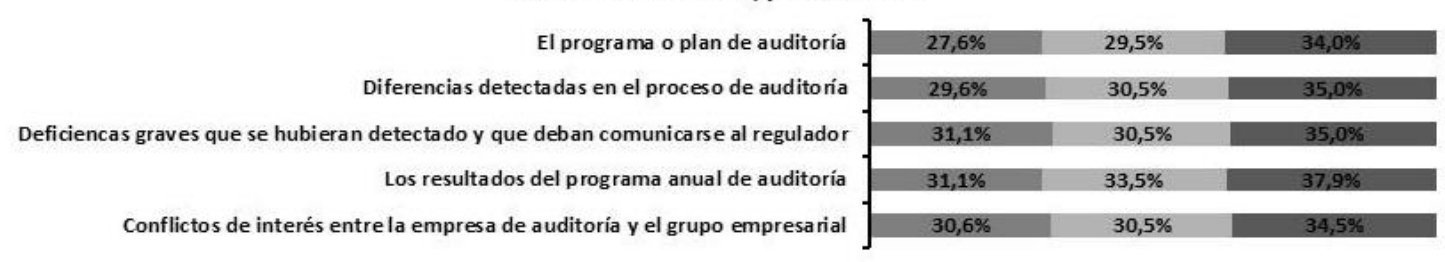

El directorio se reune al menos trimestralmente con la unidad de gestión de riesgos de la entidad o responsable de función equivalente, para analizar:

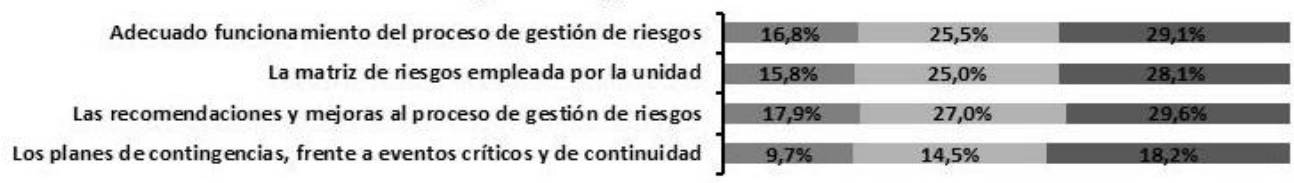

El directorio se reúne al menos trimestralmente con la unidad de auditoría interna, oficial de cumplimiento o responsable de función equivalente, para analizar:

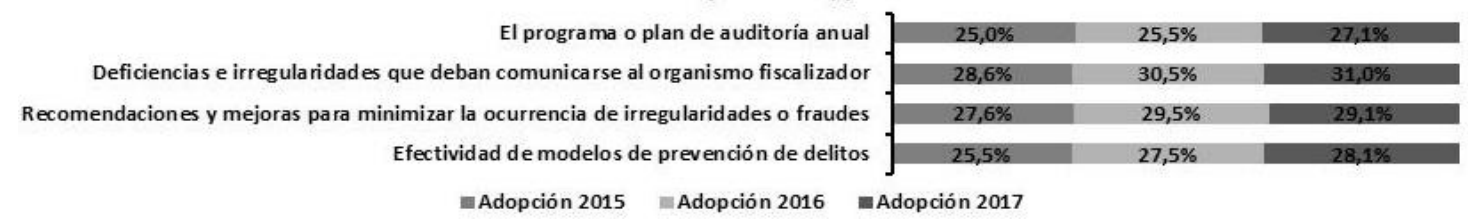

Figura №7. Grado de adopción por prácticas, principio 1.d, 1.e, 1.f.

Fuente: Elaboración Propia

Otra de las temáticas importantes atribuidas al funcionamiento del directorio se relaciona al mejoramiento continuo, área que presenta una adhesión que fluctúa el 2017 entre el 16,3\% y un $31,5 \%$, la Figura $N^{\circ} 8$, señala que, un $31,5 \%$ de las empresas tiene procedimientos formales para detectar e implementar mejoras en la organización, un 23,2\% tiene procedimientos formales en cuanto a los reuniones y tiempo de dedicación del Directorio, mientras que solo un $20,2 \%$ tiene considerado la asesoría en materia de continuidad de un experto ajeno a la sociedad.

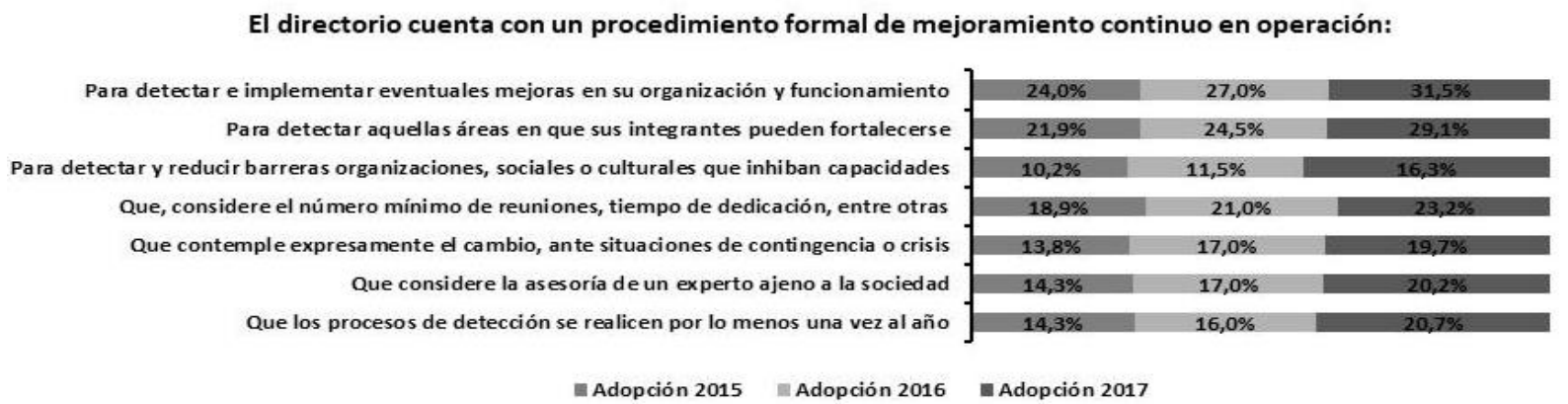

Figura $N^{` 8}$. Grado de adopción por prácticas, principio 1.j

Fuente: Elaboración Propia

Al abordar el área relacionada con los accionistas, los resultados respecto a la implementación de un procedimiento formal y en operación donde se informe distintos puntos,

"Visión de Futuro" Año 16, Volumen Nº 23 N², Julio - Diciembre 2019 - Pág. 37 - 55

URL de la Revista: http://visiondefuturo.fce.unam.edu.ar/index.php/visiondefuturo/index

URL del Documento: http://visiondefuturo.fce.unam.edu.ar/index.php/visiondefuturo/issue/view/16

ISSN 1668 - 8708 - Versión en Línea

E-mail: revistacientifica@fce.unam.edu.ar 
estos según Figura $N^{\circ} 9$ indican que solo el 5,9\% de las sociedades informa con 3 meses de antelación una referencia del candidato a Director. Lo anterior, contrasta con el 58,6\% informado a los accionistas respecto a la experiencia, profesión u oficio del candidato.

Otra recomendación importante, se refiere a si la sociedad cuenta con un departamento de relaciones con el accionista, en este punto, un 63,5\% posee este departamento, donde los accionistas aclaran sus dudas y consultas.

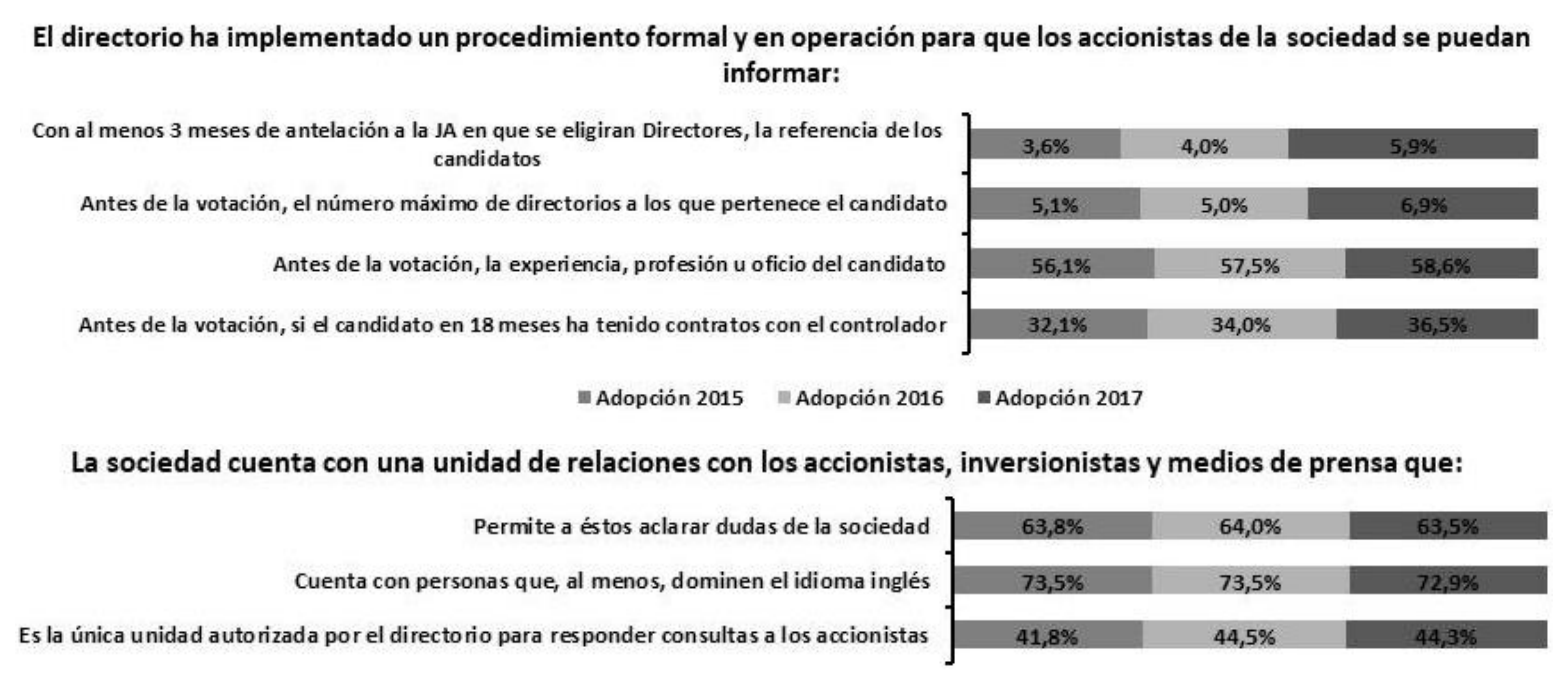

Figura $\mathrm{N}^{\circ}$ 9. Grado de adopción por prácticas, principio 2.a y 2.e

Fuente: Elaboración Propia

La tercera categoría de la normativa aborda el área de gestión de riesgos, siendo la que presenta un mayor grado de adopción, por ejemplo, de acuerdo a la Figura №10, la adopción de las prácticas relacionadas a la implementación de un proceso formal y que se encuentre en operación fluctúan entre un $66,0 \%$ y un $40,9 \%$, destacando entre otros resultados el $47,3 \%$ en la adhesión a guías, principios y directrices de gestión de riesgos en la operación de la compañía, como también el $55,7 \%$ de sociedades que posee una unidad formalizada de auditoría interna.

También, la Figura $\mathrm{N}^{\circ} 10$ aborda el canal de denuncia ético, el que se encuentra en un $68 \%$ implementado y en operación por las compañías, garantizando en un 70,4\% el anonimato del denunciante, adicionalmente un $55,2 \%$ de las sociedades señala que el denunciante puede conocer el estado de su denuncia tras la consulta. 
El directorio ha implementado un proceso formal de Gestión y Control de Riesgos el cual se encuentra en operación y que:

\begin{tabular}{|c|c|c|c|}
\hline Tiene como directrices las políticas de gestión de riesgos aprobadas por el Directorio & $46,4 \%$ & $57,0 \%$ & $59,6 \%$ \\
\hline Cuenta con una unidad de Auditoría interna o equivalente & $45,9 \%$ & $53,0 \%$ & \\
\hline Es revisado y actua lizado, al menos a nualmente & $34,7 \%$ & $45,0 \%$ & \\
\hline Considera el impacto de los riesgos de sostenibilidad económicos, sociales y a mbientales & $33,2 \%$ & $45,0 \%$ & $47,3 \%$ \\
\hline Contempla un Código de Consuta o equivalente & $56,1 \%$ & $61,5 \%$ & \\
\hline Contempla la información y capacitación permanente de todo el personal atingente & $34,2 \%$ & $39,5 \%$ & \\
\hline
\end{tabular}

El directorio ha implementado un procedimiento formal y que está en operación:

Para canalizar las denuncias por parte de su personal, cualquiera sea el vínculo contractual Que garantiza el anonimato del denunciante

Que permite al denunciante conocer el estado de su denuncia Que es puesto en conocimiento de su personal, u otra parte interesada

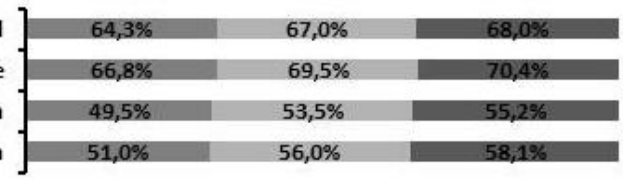

॥adopción 2015 \#dopción 2016 \#Adopción 2017

Figura $N^{\circ}$ 10. Grado de adopción por prácticas, principio 3.a y 3.b

Fuente: Elaboración Propia

Continuando, la Figura №11, muestra la autoevaluación del directorio respecto a las prácticas contenidas en la NCG №385. De acuerdo a los resultados, solo un 13,3\% de las sociedades ha sometido a revisión de un tercero ajeno a la sociedad la autoevaluación del directorio, siendo tan solo de un 6,9\% el 2017 las sociedades en los cuales sus revisores son fiscalizados o se encuentran inscritos en alguna bolsa de comercio.

La autoevaluación del directorio respecto a la adopción de las prácticas contenidas en la presente normativa:

Los que realizaron la revisión, tienen al menos 5 años de experiencia en el área Los que realizaron la revisión, son fiscalizados por la Superintendencia u organismo público

Los que realizaron la revisión, pertenecen a alguna nómina establecida por la bolsa

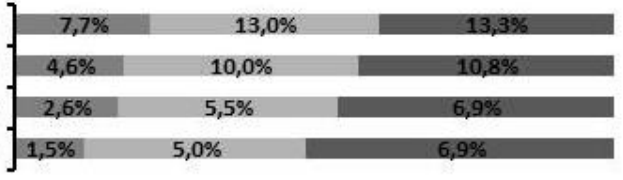

Mdopción 2015 Adopción 2016 Adopción 2017

Figura $\mathrm{N}^{\circ}$ 11. Grado de adopción por prácticas, principio 4.a

Fuente: Elaboración Propia

Finalmente, los resultados en los distintos niveles de estudio, señalan que existe un estancamiento en la adopción de la NCG №385, y ya transcurrido tres períodos de la promulgación de la normativa de GC, no existe claridad si las sociedades aumentaran significativamente su adhesión a la normativa en los futuros reportes.

\footnotetext{
“Visión de Futuro" Año 16, Volumen N²3 N², Julio - Diciembre 2019 - Pág. 37 - 55

URL de la Revista: http://visiondefuturo.fce.unam.edu.ar/index.php/visiondefuturo/index

URL del Documento: http://visiondefuturo.fce.unam.edu.ar/index.php/visiondefuturo/issue/view/16

ISSN 1668 - 8708 - Versión en Línea

E-mail: revistacientifica@fce.unam.edu.ar
} 


\section{Discusión de Resultados}

Tras ilustrar los resultados de la NCG N³85, comparativamente, no ha existido un avance significativo entre los períodos 2015 - 2017, tampoco a nivel de categorías y principios, por tanto, si el objetivo del regulador al derogar la NCG N`341 y promulgar la NCG `385 fue fortalecer la estructura de GC de las sociedades anónimas, todavía el indicador de gobernanza se clasificaría como débil e incipiente.

Entre los factores que podrían explicar esta situación, se pudiera identificar la falta de compromiso con la transparencia de parte de las empresas, al considerar poco conveniente divulgar dicha información a sus stakeholders (Moraga \& Ropero, 2018), influenciado por la alta concentración y la existencia de accionistas controladores en un número importante de sociedades anónimas chilenas (Zegers \& Consiglio, 2013).

Por otro lado, uno de los factores del por qué no se proveería información relevante al mercado, sería posiblemente porque la decisión de comunicar las prácticas es para cumplir con el regulador y no necesariamente por convicción empresarial (Godoy et al, 2018), siendo el principio Comply or Explain, promovido por la OCDE, el que permita al inversionista decidir si las razones entregadas por la entidad son suficientes y de no serlo, serán ellos los que al tomar sus decisiones de inversión considerarán la decisión de la entidad (Superintendencia de Valores y Seguros, 2015).

Finalmente existen áreas, donde el grado de adopción es preocupante, por ejemplo en responsabilidad social, mejoramiento continuo, adopción de estándares internacionales, aprobación de estructuras salariales y autoevaluación del Directorio, donde la adhesión es baja dado la importancia de las temáticas abordadas.

\section{CONCLUSION}

El estudio ha permitido establecer que, en los tres años de vigencia de la NCG N³85, el grado de adopción a las prácticas de gobierno corporativo es bajo. Además, no se percibe interés, por parte de las sociedades, en implementar un mayor número de recomendaciones dado el poco avance en la adopción en los tres años analizados.

En relación a las categorías de gobernanza, se ha identificado que existe poco interés por parte del directorio en implementar prácticas relacionadas a promover la información, participación y transparencia con los accionistas. Adicionalmente, se puede inferir que los directores no comporten la recomendación relacionada a la autoevaluación que debe realizar el directorio, por lo menos una vez al año, sobre su desempeño en materia de gobernanza corporativa. 
Por otra parte, es insuficiente la labor de supervisión que realizan los directorios en Chile, considerando la baja adopción de prácticas respecto a los procesos de monitoreo de áreas relacionadas al aseguramiento, como lo son auditoría interna, gestión de riesgos y auditoría externa. Adicionalmente, la falta de procedimientos formales relacionados al mejoramiento continuo de la compañía, y a la baja adopción de prácticas referidas a los mecanismos de capacitación del directorio, permite cuestionar si las funciones de este órgano de gobierno se están llevando de acuerdo a lo que esperan los diferentes stakeholders del mercado de valores.

También, tras los resultados, el bajo grado de adopción de la NCG №385 permite la opción de evaluar si el principio normativo Comply or Explain promovido por la OCDE para sus países miembros, pudiera no ser el mejor criterio para países con características económicas y culturales como las de Chile u otros países latinoamericanos.

En cuanto al informe, este debiera ser revisado y auditado por un tercero independiente, como ocurre con los Estados Financieros de las sociedades, debido a que la autoevaluación más que un reporte a un ente regulador, parece ser un check list donde se debe colocar Sí o No, careciendo de un proceso de auditoría o certificación que acredite la información con evidencia real.

Adicionalmente, falta un proceso de fiscalización por parte del regulador, que permita corroborar si la información declarada por las sociedades en su reporte es verosímil, sobre todo, porque la NCG N³85 dentro de sus objetivos, es brindar al inversionista mejor información para que pueda decidir si invertir o no en la sociedad.

Respecto al número de recomendaciones que establece la normativa, la baja adopción pudiera deberse a que la CMF, no ha incorporado un apéndice donde sugiere por el tamaño de la sociedad que práctica debiera adoptar, debido a que se está evaluando con la misma recomendación a empresas que cotizan en la bolsa de Nueva York, como sociedades que poseen una actividad económica local, sin mayor incidencia en el mercado de valores chileno.

Finalmente, el describir y presentar el grado de adopción de las principales empresas del mercado bursátil chileno en un período de tiempo, permitiría iniciar futuras investigaciones relacionadas a la efectividad de la propuesta realizada por el regulador y si la adhesión conlleva comparativamente futuros beneficios a las entidades en un período definido de tiempo.

\footnotetext{
"Visión de Futuro" Año 16, Volumen Nº 23 N², Julio - Diciembre 2019 - Pág. 37 - 55

URL de la Revista: http://visiondefuturo.fce.unam.edu.ar/index.php/visiondefuturo/index

URL del Documento: http://visiondefuturo.fce.unam.edu.ar/index.php/visiondefuturo/issue/view/16

ISSN 1668 - 8708 - Versión en Línea

E-mail: revistacientifica@fce.unam.edu.ar
} 


\section{REFERENCIAS}

A\&C Consultores Auditores SpA. (2019). Revisión de resultados: Implementación Norma de Carácter General N`385 de Gobiernos Corporativos. ¿Se avanza en materia de gobernanza corporativa?. Recuperado de: //www.elmostrador.cl/media/2016/07/Revision-de-resultados.-Implementacion-Normade-Caracter-General-N\%C2\%B0385-de-Gobiernos-Corporativos.pdf

Agüero, J. (2009). Gobierno Corporativo: una Aproximación al Estado del Debate. Visión de Futuro. Vol. $11\left(\mathrm{~N}^{\circ} 1\right)$.

Altamirano et al., (2019). Gobierno Corporativo e Innovación Social en el sector de la economía popular y solidaria. Uniandes Episteme. 24-48.

Anderson, et al, (2008). Estadística para Administración y Economía. México D.F., México D.F., México: CENGAGE.

Arenas, F., \& Sánchez, J. (2017). Gobiernos Corporativos: Resultados de la aplicación de la Norma de Carácter General №385. Contabilidad, Auditoría e IFRS. 83.

Briozzo, et al, (2018). Análisis del marco normativo del gobierno corporativo para la Argentina. Visión de Futuro. 33-53.

Castro, R., \& Cano, M. (2004). Buen gobierno corporativo, solución a la crisis de confianza: Cambios en la contabilidad y auditoría, aporte a los estándares internacionales, apuntes de la comparación de los casos Parmalat - Enron. Contaduría Universidad de Antoquía. 17-51.

Fundación Instituto Iberoamericano de Mercado de Valores. (2015). El Gobierno Corporativo en Iberoamérica. Madrid: Fundación Instituto Iberoamericano de Mercado de Valores. Recuperado de: http://www.iimv.org/iimv-wp-1-0/resources/uploads/2015/04/gobiernocorporativo_web.pdf

Godoy et al, (2018). Análisis del primer intento de imponer autorregulación sobre gobierno corporativo en Chile: Resultados de un Oxímoron. Revista Chile de Derecho. 179-210.

Hueso, A., \& Cascant, M. (2012). Metodología y Técnicas Cuantitativas de Investigación. Valencia-España, Editorial Universitat Politecnica de Valencia.

Iltis Consulting. (21 de 01 de 2019). Iltis. Recuperado de: https://www.iltis.cl/wpcontent/uploads/2018/07/Gobiernos-Corporativos-en-Chile-NCG-385-Julio-2018.pdf

Ministerio de Hacienda. (20 de 10 de 2009). Introduce perfeccionamiento a la normativa que regula los gobiernos corporativos de las empresas. Chile.

Moraga, H., \& Ropero, E. (2018). Gobierno Corporativo y desempeño financiero de las empresas más importantes del mercado búrsatil Chileno. Revista Venezolana de Gerencia. 145-162.

\footnotetext{
“Visión de Futuro" Año 16, Volumen N²3 N², Julio - Diciembre 2019 - Pág. 37 - 55

URL de la Revista: http://visiondefuturo.fce.unam.edu.ar/index.php/visiondefuturo/index

URL del Documento: http://visiondefuturo.fce.unam.edu.ar/index.php/visiondefuturo/issue/view/16 
Organización para la Cooperación y el Desarrollo Económico. (2004). Principios de Gobierno Corporativo. Paris.

Paul, E. (2017). El aporte del gobierno corporativo en las soluciones de los problemas actuales de la empresa moderna. Revista de Derecho. 3-25.

PwC Chile y Ese Business School Universidad de los Andres. (2016). Implementación de la Norma de Carácter General N³85: Estatus de los directorios en Chile. 1-44. Recuperado

de:https://www.pwc.com/cl/es/publicaciones/assets/2016/Estatus_de_los_directorios_en _chile_en_NCG385.ptx.pdf.

Richart-Ramón et al, (2011). Análisis de la producción científica sobre Gobierno Corporativo a través de ISI Web of Science. Revista Española de Documentación Científica. 1-23.

Rivero, P. (2005). Responsabilidad social y gobierno corporativo: Información y transparencia. Revista Asturiana de Economía, Volumen 9 (º30). Pág. 27.

Ruiz, A., \& Steinwascher, W. (2007). Gobierno Corporativo, diversificación estratégica y desempeño empresarial en México. MPRA, Paper No. 3819 (5): 1-16.

Sánchez, J., \& García-Meca, E. (2004). La influencia del gobierno corporativo en la rentabilidad de las empresas españolas. Boletín de Estudios Económicos. 305-322.

Superintendencia de Valores y Seguros. (2015). Informe Final: Fortalecimiento de Estándares de Gobierno Corporativo de las Sociedades Anónimas Abiertas. Santiago: SVS. Recuperado de: https://www.svs.cl/portal/principal/605/articles-20924_doc_pdf.pdf Superintendencia Valores y Seguros. (08 de 06 de 2015). Norma de Carácter General Nㅜ385. Recuperado de: https://www.svs.cl/normativa/ncg_385_2015.pdf

Zegers, M., \& Consiglio, J. (2013). Gobierno Corporativo en Latinoamérica: Un análisis comparativo entre Brasil, Chile, Colombia y México. Centro de Gobiernos Corporativos, Pontificia Universidad Católica de Chile. Recuperado de: http://centrogobiernocorporativo.uc.cl/Ver-documento/273-Gobierno-Corporativo-enLatinoamerica-Un-Analisis-Comparativo-entre-Brasil-Chile-Colombia-y-Mexico.html.

\section{RESÚMEN BIOGRÁFICO}

\section{Felipe Arenas Torres}

Magíster en Contabilidad y Auditoría de la Universidad de Santiago de Chile. Académico Facultad de Economía y Negocios, e integrante del Centro de Investigación y Estudios Contables de la Universidad de Talca, Chile. Línea de interés Gobiernos Corporativos y Procedimientos de Auditoría.

\footnotetext{
"Visión de Futuro" Año 16, Volumen Nº 23 N², Julio - Diciembre 2019 - Pág. 37 - 55

URL de la Revista: http://visiondefuturo.fce.unam.edu.ar/index.php/visiondefuturo/index

URL del Documento: http://visiondefuturo.fce.unam.edu.ar/index.php/visiondefuturo/issue/view/16 


\section{Roberto Mauro Campos Troncoso}

Doctor en Administración de Empresas PhD. de la Universidad Central de Nicaragua. Desde 1995 a la actualidad es académico e investigador de la Escuela de Auditoria e Ingeniería en Control de Gestión de la Facultad Economía y Negocios FEN, de la Universidad de Talca, Chile.

\section{Valentín Santander Ramírez}

Doctor Cs Humanas y Sociales, U. de Almería, España. Académico Facultad de Economía y Negocios de la Universidad de Talca, Chile. Director Escuela Auditoría e Ingeniería en Control de Gestión. Línea de interés Gestión de organizaciones, Interculturalidad en ámbito negocios, Educación del Contador Público. 